\title{
Allergies in urban versus countryside settings in Poland as part of the Epidemiology of the Allergic Diseases in Poland (ECAP) study - challenge the early differential diagnosis
}

\author{
Edyta Krzych-Fałta ${ }^{1}$, Konrad Furmańczyk ${ }^{1,2}$, Barbara Piekarska ${ }^{1}$, Aneta Tomaszewska' ${ }^{1}$, Adam Sybilski ${ }^{1,3}$, \\ Bolesław K. Samoliński ${ }^{1}$
}

${ }^{1}$ Unit of Environmental Hazard Prevention and Allergology, Faculty of Health Science, Medical University of Warsaw, Warsaw, Poland 2Department of Applied Mathematics, Warsaw University of Life Sciences, Warsaw, Poland.

${ }^{3}$ Department of Pediatrics and Neonatology, Central Clinical Hospital of the Ministry of Interior, Warsaw, Poland

Adv Dermatol Allergol 2016; XXXIII (5): 359-368

DOI: 10.5114/pdia.2016.61338

\begin{abstract}
Introduction: The incidence of allergies depends on a number of factors, including adopting an urban "western" lifestyle, genetic predispositions, and different regions of residence.

Aim: To compare the prevalence of allergic conditions (seasonal allergic rhinitis (SAR) and perennial allergic rhinitis (PAR), bronchial asthma (BA), atopic dermatitis (AD)) in a group of countryside versus urban residents in Poland. Material and methods: The prevalence of allergic conditions in urban versus countryside settings was assessed using the translated and approved questionnaire developed for international ECRHS II and ISAAC studies. Respondents were selected via random multistage sampling, with proportionate stratified sampling, and the Polish Resident Identification Number (PESEL) as the basis. A total of 18,617 respondents took part in the study. Subsequently, approximately $25 \%$ of the subjects underwent outpatient assessments: skin-prick, lung function, peak nasal inspiratory flow (PNIF) tests, as well as history-taking.

Results: Allergic rhinitis (AR) proved to be the most common condition in the entire study population. Children residing in the countryside were twice more likely to be diagnosed with $\mathrm{BA}(8.33 \%$ vs. $4 \% ; p<0.05)$. Conversely, in the adult subgroup, BA was more commonly observed in urban areas. Whereas reported symptom rates were much higher in AR and AD patients, symptomatic BA was proportionately lower with respect to the official diagnoses (underdiagnosed BA phenomenon). Atopic dermatitis was considerably more common in the metropolitan population. One factor that significantly correlated with allergic diseases was a positive family history.

Conclusions: Inhabitants of metropolitan areas are to a greater extent predisposed to allergic conditions. One factor significantly contributing to allergies is genetic predisposition. Given the scale of the problem, there is an urgent need to implement measures for early prevention and diagnosis of allergies to minimize distant health effects.
\end{abstract}

Key words: epidemiology of allergic diseases in Poland, environmental, allergy prevalence, urban versus countryside.

\section{Introduction}

During the current era of diseases of civilization, including allergies, there is an urgent need to fulfil health policies in terms of disease prevention as well as increasing health awareness to reduce the associated risk of complications, including generally low public fitness levels and healthcare system problems. Early diagnosis of allergies including skin prick tests, slgE play a fundamental role in the diagnosis of allergic diseases. An extremely interesting phenomenon which is more and more often addressed in the literature on the subject is the local IgE-dependent reaction within the nasal cavity especially in the group of patients with no signs of atopy and having specific IgE antibodies only in the na-

Address for correspondence: Edyta Krzych-Fałta, Unit of Environmental Hazard Prevention and Allergology, Faculty of Health Science, Medical University of Warsaw, 1A Banacha St, 02-097 Warsaw, Poland, phone: +48 2259910 40, fax: +48 225992042 , e-mail: e.krzych@gmail.com

Received: 15.07.2015, accepted: 2.10.2015. 
sal lavage fluid, which are not confirmed by skin tests or slgE. A nasal challenge test with an allergen, defined as a response of the mucous membrane to environmental factors, which involves the IgE-dependent reaction - is the only method in the group of diagnostic tools enabling tracking the reaction, which frequently in the differential diagnosis scheme, particularly of the allergic rhinitis, allows for the identification of a group of patients with isolated type of IgE-dependent reaction within the nasal mucous membrane. An estimated 35\% of the general population in developed countries has been diagnosed with an allergic condition, which makes allergies a modern-day epidemic and the disease of the $21^{\text {st }}$ century [1]. The growing incidence of allergies is mostly observed in the urban in comparison to the countryside population. The protective effect seen in the countryside is due to exposure to pathogens from a very young age, drinking raw (unpasteurized) milk, and not striving to achieve "sterile" living conditions. These factors have been included in the hygienic hypothesis that describes a tendency to produce Th2 lymphocytes and allergy development [2, 3]. Conversely, the high level of air pollution, heavy traffic, and urbanization observed in cities significantly contribute to developing allergic conditions.

\section{Material and methods}

The aim of this study was to assess the prevalence of allergic conditions, including allergic rhinitis (AR), bronchial asthma (BA), and atopic dermatitis (AD) in subjects residing in the countryside in comparison to the rates of those conditions in residents of the most urbanized regions in Poland. The results of the Epidemiology of the Allergic Diseases in Poland (ECAP) study conducted in the period from 2006 to 2008 not only illustrate the current condition of Poles in various regions of Poland and differences in the incidence of allergies to common environmental allergens, but also help predict the epidemiological course of allergies over the next few years.

Target study No. 6 PO5 2005 C/06572 "Initiation of the plan for prevention and early detection of allergic diseases in Poland" (ECAP) involved a population of adults aged 20-44 (standard European Community Respiratory Health Survey, ECRHS) and children aged 6-7 and 13-14 (standard International Study of Asthma and Allergies in Childhood, ISAAC, questionnaire) residing in eight of the largest Polish metropolitan areas (Gdansk, Wroclaw, Poznan, Katowice, Krakow, Lublin, Bialystok and Warsaw), and countryside areas (Zamojski and Krasnostawski counties). Subject distribution in terms of the number of urban and countryside residents was comparable (Table 1). The study design involved two main stages of field research: a survey study based on translated and approved versions of the standard ECRHS and ISAAC questionnaires and clinical (outpatient) assessments conducted according to ECRHS II standards (25\% of the respondents completed the ECAP questionnaire). Physicians established the diagnoses based on the standard criteria for the diagnosis of asthma (based on GINA, Global Strategy for Asthma Management and Prevention) and allergic rhinitis (based on ARIA, Allergic Rhinitis and its Impact on Asthma). The ECAP study was conducted in a group of 9,000 children randomly selected via the Polish Resident Identification Number (PESEL) database by the Ministry of Interior and Administration (with ISAAC questionnaires for 6-7 and 13-14 age groups) and a group of 13,500 adults (with ECRHS questionnaires for the 20-44 age

Table 1. Study group characteristics

\begin{tabular}{|c|c|c|c|c|c|c|}
\hline \multirow[t]{2}{*}{ Parameter } & \multicolumn{2}{|c|}{$6-7$ years } & \multicolumn{2}{|c|}{ 13-14 years } & \multicolumn{2}{|c|}{ Adults } \\
\hline & $n$ & $\%$ & $n$ & $\%$ & $n$ & $\%$ \\
\hline \multicolumn{7}{|c|}{ Part I: Questionnaire-based study (patient-reported symptoms) } \\
\hline Urban total & 4048 & 24.44 & 4177 & 25.22 & 8337 & 50.33 \\
\hline Urban females & 1987 & 49.08 & 2014 & 48.21 & 4936 & 59.20 \\
\hline Urban males & 2061 & 50.91 & 2163 & 51.78 & 3401 & 40.79 \\
\hline Countryside total & 462 & 22.48 & 544 & 26.47 & 1049 & 51.04 \\
\hline Countryside females & 231 & 50 & 261 & 47.97 & 582 & 55.48 \\
\hline Countryside males & 231 & 50 & 283 & 52.02 & 467 & 44.51 \\
\hline \multicolumn{7}{|c|}{ Part II: Outpatient assessment (physician's diagnosis) } \\
\hline Urban total & 1174 & 27.70 & 1149 & 27.11 & 1915 & 45.18 \\
\hline Urban females & 575 & 48.97 & 559 & 48.65 & 1168 & 60.99 \\
\hline Urban males & 599 & 51.02 & 590 & 51.34 & 747 & 39.00 \\
\hline Countryside total & 156 & 28.62 & 172 & 31.55 & 217 & 39.81 \\
\hline Countryside females & 82 & 52.56 & 80 & 46.51 & 143 & 65.89 \\
\hline Countryside males & 74 & 47.43 & 92 & 53.48 & 74 & 34.10 \\
\hline
\end{tabular}


group) in 9 regions of Poland (with Warsaw, Lublin, Bialystok, Gdansk, Poznan, Wroclaw, Katowice, Krakow representing metropolitan areas and Zamojski and Krasnostawski counties representing the countryside). The study design included two main parts. Part I: a questionnaire-based study (22,500 respondents) conducted using the Computer-Assisted Personal Interviewing (CAPI) with Personal Digital Assistant (PDA) devices. Part II: a complementary clinical assessment in 7,000 subjects including: spirometry (EasyOne 2001, Aeromedica, Warszawa) with bronchodilator challenge, skin-prick tests, peak nasal inspiratory flow (PNIF, Clement Clark International, England) before and after nasal decongestant administration, blood sampling for genetic and immune tests according to Clinical Report Form (CRF) instructions and skin-prick tests (Allergopharma; birch, grasses/cereals, wormwood, Dermatophagoides pteronyssinus and Dermatophagoides farinae), type-I molds (Botrytis cinerea, Cladosporium herbarium, Alternaria tenuis, Curvularia lunata, Fusarium moniliforme, Helminthosporium), type-Il molds (Aspergillus fumigatus, Mucom mucedo, Penicillium notatum, Pullularia pullulans, Rhizopus nigricans, Serpula lacrymans), dog, cat, hazel, alder, rye, plantain, Cladosporium herbarium, Alternaria tenuis as well as negative and positive histamine/negative controls. The following questions were used to assess reported allergy rates: question V 176 "Do you have any nasal allergies, including a runny nose caused by allergy to pollens (hay fever)?" (for AR); question V 160 "Have you ever had asthma?" (for BA); question V 191 "Have you ever had eczema (dermatitis) or any other form of skin allergy?" (for AD). The outpatientsetting diagnosis was based on the patient's history and included the following diagnoses: seasonal allergic rhinitis $(S A R)$, perennial allergic rhinitis (PAR), atopic BA, and AD. The study was approved by the Ethics Committee of the Medical University of Warsaw and the Inspector General for Personal Data Protection.

\section{Statistical analysis}

In Tables 1-3, the standard statistical analysis for contingence tables has been done. The logistic regression models were used for calculation of the risk factors with the OR risk (Tables 2, 4). For assessing the statistical significance of OR for a considered risk factor, the Wald test has been performed. $P$-value of $<0.05$ was considered to be statistically significant.

\section{Results and discussion}

\section{Part one: Questionnaire-based study (subject- reported symptoms)}

The rates of patient-reported symptoms assessed via the questionnaire-based survey showed significant discrepancies versus the rates of the corresponding clinical diagnoses based on a physician-administered questionnaire, skin-prick test results, and spirometric findings. An analysis of the survey questionnaire showed a markedly higher incidence of allergic conditions in the urban population. Allergic rhinitis was found to affect a significantly (over 1.5 times) greater proportion of the urban population versus the evaluated countryside regions. Although considerably greater proportions of $A R$ and $A D$ patients reported symptoms, symptoms of BA were reported less frequently in comparison to the respective clinical diagnoses, with BA affecting more commonly metropolitan populations. Bronchial asthma was diagnosed considerably more often in adolescents aged 13-14 and adults (Table 2). The highest proportion of reported symptoms was associated with skin lesions, including AD. Moreover, respondents from metropolitan areas, especially children aged 6-7 and 13-14, had a markedly higher risk of AD.

\section{Part two: Outpatient assessment (clinical diagnosis)}

The study, conducted in accordance with the ECRHS and ISAAC standards, revealed the greatest proportion of subjects to be affected by AR. The age groups characterized by the highest AR prevalence were adolescents (aged 13-14) and adults (aged 22-44). Interestingly, perennial $A R$ was shown to be most prevalent in urban populations, whereas countryside residents, apart from 13-14-year-old adolescents, most often suffered from SAR. The second most prevalent allergic condition in the Polish population was BA. It was twice as prevalent in 6-7-year-olds and slightly more in 13-14-year-olds residing in the countryside versus their urban counterparts. Conversely, in the adult population, BA was more common in urban residents. Atopic disease was markedly more common in populations from urban areas. In fact, $A D$ affected over 3.5 times more 6-7-year-old children and two times more 13-14-year-old adolescents in cities, in comparison with their peers living in the countryside. Any region-related differences in $A D$ symptom rates in adults were not statistically significant.

\section{Positive skin-prick test rates}

The subpopulation in which skin-prick tests were conducted most often and yielded most pronounced reactions were residents of metropolitan areas (Table 3). Seasonal allergens were the cause of more cases of allergy than perennial ones. The youngest evaluated age group tested positive mostly for the following allergens: grass/cereals, house dust mites, rye, birch, and cat, with these allergens also producing the most pronounced skin reactions. Positive skin-prick tests in the group aged 13-14 in cities were mostly due to the same allergens as in the youngest evaluated group and additionally included wormwood, birch, and dog allergens. The number of allergens producing a positive reaction increased with age (with ribleaf, alder, and hazel, yielding highly positive skin-prick tests). Conversely, the most prevalent allergies 
Table 2. Allergy prevalence stratified by region

Part I: Questionnaire-based study (patient-reported symptoms)

\begin{tabular}{|c|c|c|c|c|c|c|c|c|c|c|c|c|}
\hline \multirow{2}{*}{\multicolumn{2}{|c|}{ Parameter }} & \multicolumn{4}{|c|}{$6-7$ years } & \multicolumn{3}{|c|}{$13-14$ years } & \multicolumn{4}{|c|}{ Adults } \\
\hline & & \multicolumn{2}{|c|}{$n$} & \multicolumn{2}{|l|}{$\%$} & \multicolumn{2}{|l|}{$n$} & $\%$ & \multicolumn{2}{|r|}{$n$} & \multicolumn{2}{|c|}{$\%$} \\
\hline \multicolumn{2}{|l|}{ Urban AR } & \multicolumn{2}{|c|}{980} & \multicolumn{2}{|c|}{24.30} & \multicolumn{2}{|l|}{1051} & 25.20 & \multicolumn{2}{|c|}{1833} & \multicolumn{2}{|c|}{22.02} \\
\hline \multicolumn{2}{|c|}{ Countryside AR } & \multicolumn{2}{|c|}{85} & \multicolumn{2}{|c|}{18.39} & \multicolumn{2}{|l|}{109} & 20.03 & \multicolumn{2}{|r|}{134} & \multicolumn{2}{|c|}{12.77} \\
\hline \multicolumn{2}{|l|}{ Urban BA } & \multicolumn{2}{|c|}{163} & \multicolumn{2}{|c|}{4.04} & \multicolumn{2}{|l|}{255} & 6.13 & \multicolumn{2}{|r|}{331} & \multicolumn{2}{|c|}{3.98} \\
\hline \multicolumn{2}{|c|}{ Countryside BA } & \multicolumn{2}{|c|}{14} & \multicolumn{2}{|c|}{3.05} & \multicolumn{2}{|l|}{18} & 3.32 & \multicolumn{2}{|r|}{20} & \multicolumn{2}{|c|}{1.90} \\
\hline \multicolumn{2}{|l|}{ Urban AD } & \multicolumn{2}{|c|}{1932} & 47.78 & & 2907 & & 40.92 & & 2907 & 34.9 & \\
\hline Countryside & $A D$ & 13 & 34 & 29.06 & & 136 & & 25 & & 252 & 24.0 & \\
\hline Parameter & & $6-7$ ye & ears & & & $13-14 y$ & years & & & Adult & & \\
\hline & OR & $95 \%$ & $6 \mathrm{Cl}$ & $P$-value & OR & $95 \%$ & $6 \mathrm{Cl}$ & $P$-value & OR & $95 \%$ & $\mathrm{Cl}$ & $P$-value \\
\hline AR & 1.438596 & 1.124761 & 1.84 & $<0.05$ & 1.333333 & 1.068663 & 1.663554 & $<0.05$ & 1.887574 & 1.563195 & 2.279265 & $<0.05$ \\
\hline BA & 1.347222 & 0.773689 & 2.345913 & $<0.05$ & 2.06383 & 1.268632 & 3.357471 & $<0.05$ & 2.041667 & 1.294081 & 3.221129 & $<0.05$ \\
\hline$A D$ & 2.259947 & 1.831333 & 2.788875 & $<0.05$ & 2.084746 & 1.700623 & 2.555631 & $<0.05$ & 1.705128 & 1.469549 & 1.978473 & $<0.05$ \\
\hline
\end{tabular}

Part II: Outpatient assessment (physician's diagnosis)

\begin{tabular}{|c|c|c|c|c|c|c|c|c|c|c|c|c|}
\hline \multirow{2}{*}{\multicolumn{2}{|c|}{ Parameter }} & \multicolumn{4}{|c|}{$6-7$ years } & \multicolumn{3}{|c|}{$13-14$ years } & \multicolumn{4}{|c|}{ Adults } \\
\hline & & \multicolumn{2}{|c|}{$n$} & \multicolumn{2}{|l|}{$\%$} & \multicolumn{2}{|l|}{$n$} & $\%$ & \multicolumn{2}{|r|}{$n$} & \multicolumn{2}{|l|}{$\%$} \\
\hline \multicolumn{2}{|l|}{ Urban SAR } & \multicolumn{2}{|c|}{125} & \multicolumn{2}{|c|}{10.64} & \multicolumn{2}{|l|}{167} & 14.53 & \multicolumn{2}{|r|}{284} & \multicolumn{2}{|c|}{14.83} \\
\hline \multicolumn{2}{|c|}{ Countryside SAR } & \multicolumn{2}{|c|}{26} & \multicolumn{2}{|c|}{16.66} & \multicolumn{2}{|l|}{22} & 12.79 & \multicolumn{2}{|r|}{36} & \multicolumn{2}{|c|}{16.58} \\
\hline \multicolumn{2}{|l|}{ Urban PAR } & \multicolumn{2}{|c|}{157} & \multicolumn{2}{|c|}{13.37} & \multicolumn{2}{|l|}{194} & 16.88 & \multicolumn{2}{|r|}{303} & \multicolumn{2}{|c|}{15.82} \\
\hline \multicolumn{2}{|c|}{ Countryside PAR } & \multicolumn{2}{|c|}{16} & \multicolumn{2}{|c|}{10.25} & \multicolumn{2}{|l|}{26} & 15.11 & \multicolumn{2}{|r|}{29} & \multicolumn{2}{|c|}{13.36} \\
\hline Urban BA & & 4 & & 4.00 & & 61 & & 5.30 & & 89 & 4.64 & \\
\hline Countryside & $B A$ & 1 & & 8.33 & & 11 & & 6.39 & & 6 & 2.76 & \\
\hline Urban AD & & 11 & & 9.54 & & 111 & & 9.66 & & 68 & 3.55 & \\
\hline Countryside & $A D$ & 4 & & 2.56 & & 8 & & 4.65 & & 8 & 3.68 & \\
\hline Parameter & & $6-7$ ye & ars & & & $13-14 y$ & years & & & Adult & & \\
\hline & OR & $95 \%$ & $6 \mathrm{Cl}$ & $P$-value & OR & $95 \%$ & $6 \mathrm{Cl}$ & $P$-value & OR & $95 \%$ & $\mathrm{Cl}$ & $P$-value \\
\hline SAR & 0.603437 & 0.38089 & 0.955992 & $<0.05$ & 1.180995 & 0.74388 & 1.902092 & $>0.05$ & 0.861592 & 0.589662 & 1.258926 & $>0.05$ \\
\hline PAR & 1.344828 & 0.780675 & 2.316664 & $>0.05$ & 1.160643 & 1.268632 & 3.357471 & $<0.05$ & 1.274725 & 0.846111 & 1.920462 & $>0.05$ \\
\hline BA & 0.479167 & 0.253073 & 0.907251 & $<0.05$ & 0.824561 & 0.424887 & 1.600192 & $>0.05$ & 1.701754 & 0.735479 & 3.937526 & $>0.05$ \\
\hline$A D$ & 3.592593 & 1.306233 & 9.880873 & $<0.05$ & 2.111111 & 1.011114 & 4.407803 & $<0.05$ & 1 & 0.474057 & 2.109452 & $>0.05$ \\
\hline
\end{tabular}

$A D$-atopic dermatitis, $A R$ - allergic rhinitis, $B A$ - bronchial asthma, $P A R$ - perennial allergic rhinitis, SAR-seasonal allergic rhinitis.

were those to non-seasonal allergens, i.e. D. pteronyssinus, D. farinae, and dog. Moreover, an increased rate of allergies to wormwood and grasses/cereals was observed in adult residents of the countryside/urban areas.

\section{Factors contributing to allergic conditions} in a given population

Genetic relations were found to be one of the significant environmental-social risk factors for allergy development (Table 4). The observed risk of developing both AR and BA was nearly two times higher in subjects whose siblings and/or father were affected. Moreover, the risk of $A D$ in subjects whose paternal grandfather was affected was over two-fold higher in metropolitan residents and over 5-fold higher in the countryside. Income below $€ 1,000$ in urban areas was also associated with a higher risk of developing PAR, in comparison to that in the countryside.

The currently observed increase in the incidence of allergic conditions raises concern; there are an estimated 4 million Poles suffering from BA, and one in four residents has an allergic condition, with approximately $20 \%$ of the study population receiving inadequate treatment (based on the ECAP Report). A number of single-center 
Table 3. Proportion of positive skin-prick tests stratified by the region of residence

\begin{tabular}{|c|c|c|c|c|c|c|c|c|c|c|c|c|c|c|c|c|c|c|}
\hline \multirow[t]{3}{*}{ Parameter } & \multicolumn{6}{|c|}{$6-7$ years } & \multicolumn{6}{|c|}{$13-14$ years } & \multicolumn{6}{|c|}{ Adults } \\
\hline & \multicolumn{2}{|c|}{$\geq 3 \mathrm{~mm}$} & \multicolumn{2}{|c|}{$3-5 \mathrm{~mm}$} & \multicolumn{2}{|c|}{$\geq 6 \mathrm{~mm}$} & \multicolumn{2}{|c|}{$\geq 3 \mathrm{~mm}$} & \multicolumn{2}{|c|}{$3-5 \mathrm{~mm}$} & \multicolumn{2}{|c|}{$\geq 6 \mathrm{~mm}$} & \multicolumn{2}{|c|}{$\geq 3 \mathrm{~mm}$} & \multicolumn{2}{|c|}{$3-5 \mathrm{~mm}$} & \multicolumn{2}{|c|}{$\geq 6 \mathrm{~mm}$} \\
\hline & $n$ & $\%$ & $n$ & $\%$ & $n$ & $\%$ & $n$ & $\%$ & $n$ & $\%$ & $n$ & $\%$ & $n$ & $\%$ & $n$ & $\%$ & $n$ & $\%$ \\
\hline \multicolumn{19}{|l|}{ Urban areas: } \\
\hline Birch & 117 & 9.9 & 85 & 7.2 & 32 & 2.7 & 192 & 16.7 & 123 & 10.7 & 69 & 6.0 & 327 & 17.0 & 159 & 8.3 & 168 & 8.7 \\
\hline Grasses/cereals & 188 & 16.0 & 121 & 10.3 & 67 & 5.7 & 299 & 26.0 & 148 & 12.8 & 151 & 13.1 & 436 & 22.7 & 199 & 10.3 & 237 & 12.3 \\
\hline Wormwood & 89 & 7.5 & 76 & 6.4 & 13 & 1.1 & 202 & 17.5 & 164 & 14.2 & 38 & 3.3 & 388 & 20.2 & 268 & 13.9 & 120 & 6.2 \\
\hline $\begin{array}{l}\text { Dermatophagoides } \\
\text { pteronyssinus }\end{array}$ & 177 & 15.0 & 121 & 10.3 & 56 & 4.7 & 312 & 27.1 & 163 & 14.1 & 149 & 12.9 & 475 & 24.8 & 285 & 14.8 & 190 & 9.9 \\
\hline Type I molds & 73 & 6.2 & 51 & 4.3 & 22 & 1.8 & 141 & 12.2 & 103 & 8.9 & 38 & 3.3 & 162 & 8.4 & 128 & 6.6 & 34 & 1.7 \\
\hline Type II molds & 48 & 4.0 & 47 & 4.0 & 1 & 0.1 & 63 & 5.4 & 59 & 5.1 & 4 & 0.3 & 149 & 7.7 & 134 & 6.9 & 15 & 0.7 \\
\hline Dog & 68 & 5.7 & 65 & 5.5 & 3 & 0.2 & 85 & 7.3 & 76 & 6.6 & 9 & 0.7 & 203 & 10.6 & 189 & 9.8 & 14 & 0.7 \\
\hline Cat & 96 & 8.1 & 73 & 6.2 & 23 & 1.9 & 181 & 15.7 & 128 & 11.1 & 53 & 4.6 & 281 & 14.6 & 185 & 9.6 & 96 & 5.0 \\
\hline $\begin{array}{l}\text { Dermatophagoides } \\
\text { farinae }\end{array}$ & 168 & 14.3 & 114 & 9.7 & 54 & 4.5 & 280 & 24.3 & 154 & 13.4 & 126 & 10.9 & 427 & 22.2 & 278 & 14.5 & 149 & 7.7 \\
\hline Hazel & 78 & 6.6 & 51 & 4.3 & 27 & 2.2 & 142 & 12.3 & 94 & 8.1 & 48 & 4.1 & 278 & 14.5 & 156 & 8.1 & 122 & 6.3 \\
\hline Alder & 72 & 6.1 & 53 & 4.5 & 19 & 1.6 & 132 & 11.4 & 96 & 8.3 & 36 & 3.1 & 275 & 14.3 & 179 & 9.3 & 96 & 5.0 \\
\hline Rye & 127 & 10.8 & 79 & 6.7 & 48 & 4.0 & 216 & 18.7 & 126 & 10.9 & 90 & 7.8 & 364 & 19.0 & 184 & 9.6 & 180 & 9.3 \\
\hline Ribleaf & 49 & 4.1 & 47 & 4.0 & 2 & 0.1 & 98 & 8.5 & 80 & 6.9 & 18 & 1.5 & 207 & 10.8 & 186 & 9.7 & 21 & 1.0 \\
\hline $\begin{array}{l}\text { Cladosporium } \\
\text { herbarum }\end{array}$ & 34 & 2.8 & 30 & 2.5 & 4 & 0.3 & 53 & 4.6 & 51 & 4.4 & 2 & 0.1 & 121 & 6.3 & 113 & 5.9 & 8 & 0.4 \\
\hline Alternaria tenuis & 98 & 8.3 & 70 & 5.9 & 28 & 2.3 & 123 & 10.7 & 77 & 6.7 & 46 & 4.0 & 130 & 6.7 & 98 & 5.1 & 32 & 1.6 \\
\hline \multicolumn{19}{|l|}{ Countryside: } \\
\hline Birch & 25 & 16.0 & 24 & 15.3 & 1 & 0.6 & 20 & 11.6 & 19 & 11.0 & 1 & 0.5 & 32 & 14.7 & 32 & 14.7 & 0 & 0 \\
\hline Grasses/cereals & 29 & 18.5 & 28 & 17.9 & 1 & 0.6 & 26 & 15.1 & 23 & 13.3 & 3 & 1.7 & 43 & 19.8 & 36 & 16.5 & 7 & 3.2 \\
\hline Wormwood & 28 & 17.9 & 28 & 17.9 & 0 & 0 & 25 & 14.5 & 25 & 14.5 & 0 & 0 & 40 & 18.4 & 38 & 17.5 & 2 & 0.9 \\
\hline $\begin{array}{l}\text { Dermatophagoides } \\
\text { pteronyssinus }\end{array}$ & 35 & 22.4 & 31 & 19.8 & 4 & 2.5 & 52 & 30.2 & 41 & 23.8 & 11 & 6.3 & 66 & 30.4 & 57 & 26.2 & 9 & 4.1 \\
\hline Type I molds & 18 & 11.5 & 17 & 10.8 & 1 & 0.6 & 14 & 8.1 & 14 & 8.1 & 0 & 0 & 27 & 12.4 & 27 & 12.4 & 0 & 0 \\
\hline Type II molds & 23 & 4.0 & 23 & 4.0 & 0 & 0.1 & 17 & 5.4 & 17 & 5.1 & 0 & 0.3 & 30 & 7.7 & 30 & 6.9 & 0 & 0.7 \\
\hline Dog & 33 & 21.1 & 33 & 21.1 & 0 & 0 & 23 & 13.3 & 23 & 13.3 & 0 & 0 & 29 & 13.3 & 29 & 13.3 & 0 & 0 \\
\hline Cat & 21 & 13.4 & 20 & 12.8 & 1 & 0.6 & 13 & 7.5 & 11 & 6.3 & 2 & 1.1 & 22 & 10.1 & 20 & 9.2 & 2 & 0.9 \\
\hline $\begin{array}{l}\text { Dermatophagoides } \\
\text { farinae }\end{array}$ & 31 & 14.3 & 29 & 9.7 & 2 & 4.5 & 32 & 24.3 & 28 & 13.4 & 4 & 10.9 & 51 & 22.2 & 44 & 14.5 & 7 & 7.7 \\
\hline Hazel & 18 & 11.5 & 18 & 11.5 & 0 & 0 & 20 & 11.6 & 19 & 11.0 & 1 & 0.5 & 21 & 9.6 & 20 & 9.2 & 1 & 0.4 \\
\hline Alder & 19 & 12.1 & 19 & 12.1 & 0 & 0 & 16 & 9.3 & 15 & 8.7 & 1 & 0.5 & 18 & 8.2 & 17 & 7.8 & 1 & 0.4 \\
\hline Rye & 22 & 14.1 & 22 & 14.1 & 0 & 0 & 20 & 11.6 & 19 & 11.0 & 1 & 0.5 & 26 & 11.9 & 20 & 10.1 & 4 & 1.8 \\
\hline Ribleaf & 18 & 11.5 & 18 & 11.5 & 0 & 0 & 18 & 10.4 & 18 & 10.4 & 0 & 0 & 22 & 10.1 & 21 & 9.6 & 1 & 0.4 \\
\hline $\begin{array}{l}\text { Cladosporium } \\
\text { herbarum }\end{array}$ & 16 & 10.2 & 16 & 10.2 & 0 & 0 & 14 & 8.1 & 14 & 8.1 & 0 & 0 & 15 & 6.9 & 15 & 6.9 & 0 & 0 \\
\hline Alternaria tenuis & 19 & 12.1 & 18 & 11.5 & 1 & 0.6 & 10 & 5.8 & 9 & 5.2 & 1 & 0.5 & 14 & 6.4 & 14 & 6.4 & 0 & 0 \\
\hline
\end{tabular}


Table 4. Allergy provoking factors stratified by regions

\begin{tabular}{|c|c|c|c|c|c|c|c|c|c|c|c|c|}
\hline \multirow[t]{2}{*}{ Variable } & \multicolumn{3}{|c|}{ SAR } & \multicolumn{3}{|c|}{ PAR } & \multicolumn{3}{|c|}{ Asthma } & \multicolumn{3}{|c|}{$A D$} \\
\hline & OR & $95 \% \mathrm{Cl}$ & $P$-value & OR & $95 \% \mathrm{Cl}$ & $P$-value & OR & $95 \% \mathrm{Cl}$ & $P$-value & OR & $95 \% \mathrm{Cl}$ & $P$-value \\
\hline \multicolumn{13}{|l|}{ Urban areas: } \\
\hline \multicolumn{13}{|l|}{ Gender: } \\
\hline $\begin{array}{l}\text { Females } \\
\text { Males reference }\end{array}$ & 0.99 & $0.83-1.18$ & $>0.05$ & 0.70 & $0.59-0.83$ & $<0.05$ & 0.59 & $0.44-0.79$ & $<0.05$ & 1.14 & $0.89-1.45$ & $>0.05$ \\
\hline \multicolumn{13}{|l|}{ Income: } \\
\hline \multicolumn{13}{|c|}{ Below €275 reference level } \\
\hline$€ 275-€ 1000$ & 0.96 & $0.68-1.38$ & $>0.05$ & 1.45 & $0.99-2.12$ & $>0.05$ & 1.61 & $0.80-3.23$ & $>0.05$ & 0.74 & $0.47-1.16$ & $>0.05$ \\
\hline$>€ 1000$ & 1.43 & $0.96-2.12$ & $>0.05$ & 1.57 & $1.03-2.40$ & $<0.05$ & 1.98 & $0.92-4.23$ & $>0.05$ & 1.07 & $0.64-1.78$ & $>0.05$ \\
\hline \multicolumn{13}{|l|}{ Education: } \\
\hline \multicolumn{13}{|c|}{ Primary school reference level: } \\
\hline High school & 1.42 & $0.55-3.66$ & $>0.05$ & 0.70 & $0.33-1.44$ & $>0.05$ & 2.66 & $0.35-19.73$ & $3>0.05$ & 1.40 & $0.18-10.58$ & $>0.05$ \\
\hline $\begin{array}{l}\text { University/ } \\
\text { college }\end{array}$ & 1.62 & $0.62-4.19$ & $>0.05$ & 0.64 & $0.31-1.34$ & $>0.05$ & 1.83 & $0.24-13.80$ & $>0.05$ & 1.76 & $0.23-13.31$ & $>0.05$ \\
\hline \multicolumn{13}{|c|}{ Allergies in the family: } \\
\hline Mother & 1.17 & $0.92-1.48$ & $>0.05$ & 1.41 & $1.13-1.75$ & $<0.05$ & 1.26 & $0.87-1.83$ & $>0.05$ & 2.27 & $1.73-2.98$ & $<0.05$ \\
\hline Father & 1.68 & $1.31-2.17$ & $<0.05$ & 1.71 & $1.34-2.18$ & $<0.05$ & 1.54 & $1.02-2.31$ & $<0.05$ & 2.29 & $1.68-3.12$ & $<0.05$ \\
\hline Siblings & 1.59 & $1.29-1.96$ & $<0.05$ & 1.11 & $0.90-1.37$ & $>0.05$ & 1.46 & $1.04-2.04$ & $<0.05$ & 1.45 & $1.09-1.92$ & $<0.05$ \\
\hline $\begin{array}{l}\text { Maternal } \\
\text { grandparents }\end{array}$ & 1.19 & $0.83-1.68$ & $>0.05$ & 1.11 & $0.79-1.56$ & $>0.05$ & 1.29 & $0.75-2.23$ & $>0.05$ & & No data & \\
\hline $\begin{array}{l}\text { Paternal } \\
\text { grandparents }\end{array}$ & 1.17 & $0.73-1.89$ & $>0.05$ & 1.45 & $0.95-2.22$ & $>0.05$ & 3.13 & $1.84-5.33$ & $<0.05$ & 2.45 & $1.50-4.01$ & $<0.05$ \\
\hline \multicolumn{13}{|l|}{ Countryside: } \\
\hline \multicolumn{13}{|l|}{ Gender: } \\
\hline $\begin{array}{l}\text { Females } \\
\text { Males reference }\end{array}$ & 1.12 & $0.69-1.79$ & $>0.05$ & 0.68 & $0.41-1.13$ & $>0.05$ & 0.43 & $0.20-0.93$ & $<0.05$ & & No data & \\
\hline \multicolumn{13}{|l|}{ Income: } \\
\hline \multicolumn{13}{|c|}{ Below €275 reference level: } \\
\hline$€ 275-€ 1000$ & 0.89 & $0.52-1.51$ & $>0.05$ & 0.82 & $0.46-1.44$ & $>0.05$ & 0.51 & $0.21-1.20$ & $>0.05$ & 1.81 & $0.68-4.76$ & $>0.05$ \\
\hline$>€ 1000$ & 1.37 & $0.14-12.65$ & $>0.05$ & 1.57 & $0.17-14.57$ & $>0.05$ & Samp & le size too & small & Samp & ple size too $\mathrm{s}$ & small \\
\hline \multicolumn{13}{|l|}{ Education: } \\
\hline \multicolumn{13}{|c|}{ Primary school reference } \\
\hline High school & 4.14 & $0.52-32.92$ & $>0.05$ & 0.93 & $0.24-3.57$ & $>0.05$ & Samp & ple size too $s$ & small & Samp & ple size too s & small \\
\hline $\begin{array}{l}\text { University/ } \\
\text { college }\end{array}$ & 7 & $0.78-62.52$ & $>0.05$ & 0.47 & $0.07-3.13$ & $>0.05$ & Samp & ble size too & small & Samp & ple size too s & small \\
\hline \multicolumn{13}{|c|}{ Allergies in the family: } \\
\hline Mother & 0.45 & $0.13-1.50$ & $>0.05$ & 0.45 & $0.13-1.50$ & $>0.05$ & 0.78 & $0.18-3.40$ & $>0.05$ & & No data & \\
\hline Father & 1.22 & $0.41-3.66$ & $>0.05$ & 1.22 & $0.41-3.66$ & $>0.05$ & 1.46 & $0.32-6.49$ & $>0.05$ & 2.31 & $0.50-10.57$ & $>0.05$ \\
\hline Siblings & 1.24 & $0.60-2.57$ & $>0.05$ & 1.24 & $0.60-2.57$ & $>0.05$ & 1.51 & $0.55-4.11$ & $>0.05$ & & No data & \\
\hline $\begin{array}{l}\text { Maternal } \\
\text { grandparents }\end{array}$ & 0.83 & $0.10-6.75$ & $>0.05$ & 0.83 & $0.10-6.75$ & $>0.05$ & 2.18 & $0.26-18.06$ & $6>0.05$ & & No data & \\
\hline $\begin{array}{l}\text { Paternal } \\
\text { grandparents }\end{array}$ & 0.55 & $0.07-4.29$ & $>0.05$ & 0.55 & $0.07-4.29$ & $>0.05$ & 1.44 & $0.18-11.50$ & $>0.05$ & 5.19 & $1.07-25.16$ & $<0.05$ \\
\hline
\end{tabular}


studies in highly industrialized countries show that SAR sufferers constitute approximately $32.7 \%$ of the population aged $18-22$ years in Japan, $42 \%$ of 9 -year-old children in the USA, and an estimated $29 \%$ of respondents in the 5-59-year age range in the UK [4-6]. This trend of growing allergy rates can be mostly attributed to air pollution and higher prevalence of contact allergens, especially in the case of AD. Factors such as infections or exposure to endotoxins may have a protective effect or provoke the development of AD. The increased incidence of allergies in urban areas is associated with a number of factors responsible for the significant differences versus the countryside. The influence of environmental factors is mixed (complex, overlapping effects). Typically, the human body is exposed to multiple factors of various concentrations or intensities. Effects of such exposure depend not only on qualitative and quantitative composition of these factors, but also on the potential for their interaction.

Attempts to determine the significance of the individual factors have often yielded inconclusive results and effects of a certain factor confirmed in one study have not been confirmed in another. These discrepancies may be due to divergent criteria for diagnosing allergies used by different authors, as well as different study designs. There are many environmental factors that contribute to developing allergies, including the phenomenon of thermal inversion (air temperature increases with increasing altitude, which leads to a high concentration of pollens and airborne chemical compounds), different seasons (spring and summer are the periods of most intense pollen emissions), changes in weather during the pollen season (thunderstorms may induce hydration of pollen grains and fragmentation of various pollen particles, which increases the frequency of asthma attacks), humidity, temperature, and wind speed.

There are a number of epidemiology studies demonstrating a close association between the incidence of allergies and the concentration of air pollutants [7-11] due to energy consumption and exhaust emissions [12, 13]. Air pollution is a serious environmental problem both in the developed and developing countries [11]. The most dangerous pollutants include coarse particulate matter (PM10), fine particulate matter (PM2.5), nitrogen oxides (NOX), ozone, and sulfur oxides (SOx) [11, 12, 14]. Exposure of a grass pollen to higher $\mathrm{NO}_{2}$ and ozone levels leads to increased release of allergen-laden pollen grains, thus raising the pollen's proinflammatory potential. Exposure to NOx, SOx, and ozone may damage the respiratory mucosa and disrupt the mucociliary apparatus, which increases the risk of asthmatic or allergic rhinitis exacerbations. Additional factors, i.e. cigarette smoke or Diesel exhaust fumes, may aggravate allergic reactions [2, 3].

Polish studies conducted as part of the ECAP project demonstrated that $A R$ is an allergic condition that shows most significant differences by region (city vs. countryside). The most common allergies to environmental and contact factors in the order of decreasing prevalence are as follows: $A R, B A$, and $A D$. Seasonal allergic rhinitis in the 6-7-year-old children was diagnosed in the order of decreasing prevalence in Warsaw (20.8\%), Poznan (17\%), Gdansk (15.3\%), Bialystok (10.7\%), Krakow (9.2\%), and Wroclaw (6.1\%). Perennial allergic rhinitis in this age group was diagnosed in the order of decreasing prevalence in Poznan (22.6\%), Warsaw (16.7\%), Gdansk (13\%), Wroclaw (11.4\%), Bialystok (10\%), and Krakow (2.7\%); in comparison to its $16.0 \%$ diagnosis rate in the countryside [1]. One analysis by Tomaszewska et al. conducted (as part of the ECAP study) in a smaller population of one voivodship, the city of Lublin $(n=2,422)$ and the countryside represented by the Zamojski and Krasnostawski counties ( $n=2,055)$ revealed $27.9 \%$ of the evaluated countryside population and $30.4 \%$ of the metropolitan population to be suffering from AR (SAR and PAR) $(p<$ 0.05). Bronchial asthma was found in $14.5 \%$ of urban residents and in $10.1 \%$ of countryside residents $(p<0.05)$ [15, 16]. These findings are similar to those reported by Komorowski in a study on the incidence of BA in the Polish population, which showed clear differences between the evaluated regions. Physician-diagnosed BA was observed in the following proportions of the study population: $19.5 \%$ in Poznan, $14.5 \%$ in Lublin, $12 \%$ in Gdansk, $10.6 \%$ in Krakow, 9.1\% in Warsaw, $8.1 \%$ in Katowice, and $6.0 \%$ in Bialystok [17]. Interestingly, the rates of patient-reported symptoms of AR or AD proved to be higher than the rates of these conditions confirmed with an official diagnosis. Moreover, as much as $66.9 \%$ of the patients had been unaware of their disease (underdiagnosed BA). KupryśLipińska et al. showed significantly higher rates of BA in the population of children in the evaluated urban ( $n=$ $482)$ and countryside $(n=469)$ regions, of $18.4 \%$ and $6 \%$, respectively [18]. Seasonal allergic rhinitis was diagnosed in $16.1 \%$ and $6.7 \%$ of the general population in the urban and countryside regions, respectively. The proportion of $\mathrm{AD}$ diagnoses was much higher in the countryside than in the city (11.9\% vs. $10.2 \%)$. The proportions of BA and SAR diagnoses were equal at approximately $13.2 \%$ in the group of evaluated adults in urban areas [6]. The extent of the problem is illustrated by the increasing trend in BA incidence observed in children participating in the ISAAC study in large metropolitan areas in Indonesia (4.1-2.1\% of children aged 6-7 and 13-14), in Costa Rica (32.1\% of 6-7-year-olds), in the UK (32.2\% of 13-14-year-olds). The ECRHS study additionally demonstrated the development of AD in adult populations [18].

Moreover, multicenter epidemiological studies (e.g. by Blair and Settipane) showed a higher incidence of AR symptoms in patients with BA (28-78\%) than in individuals without BA symptoms (5-20\%) [19, 20]. A breakthrough in AR and BA co-occurrence came with the ECRHS-questionnaire-based study conducted by Ley- 
naert et al. in 90,478 subjects in Spain, the UK, Australia, France, the US, Italy, and the Benelux countries. The authors demonstrated BA without $A R$ in $1-6 \%$ of respondents, BA co-occurring with AR symptoms in 7.6-22.6\% of respondents, AR without BA in 10.5-36.2\%, and AR COoccurring with BA symptoms in $50.0-77.1 \%$ of respondents $(O R=8)$ [21]. The presented study results (Figure 1 ) clearly show that the risk of BA co-occurrence in AR patients, especially those with PAR, both in the countryside and in urban areas is ten times higher in the evaluated groups and over three times higher in patients (especially female patients) with AD. A review of the available literature demonstrated that children diagnosed with $A D$ are 2.14-fold more prone to $B A$ than children without $A D$. Moreover, $A D$ in conjunction with allergies to common environmental allergens is a significant marker of the allergic march.

The highest prevalence of AD has been observed in the Scandinavian countries (15.6-22.3\%) followed by the UK (10.6-16\%), Lithuania (1.8-3\%), and Albania (2-3.7\%) [22]. In adult respondents, the highest rates of AD were found in Estonia (17.6\%), followed by the Scandinavian countries (8-9\%), the UK (8.1\%), and Switzerland (2.2\%)
[23]. Polish studies show 2.0-22.6\% (mean 9\%) of respondents diagnosed with $A D$, with considerably more 6-7-year-olds and 13-14-year-olds affected (3.5-fold and 2 -fold, respectively) [24]. There were no statistically significant differences in terms of the region of residence in adults with AD. Different regions of Poland showed different AD diagnosis rates (Hanifin and Rajka criteria): in Poznan $16.76 \%$, in Katowice $7.18 \%$, in Lublin 6.98\%, in Wroclaw 6.38\%, in Warsaw 6.31\%, in Gdansk 5.95\%, in Bialystok $4.42 \%$, and in Krakow $2.02 \%$. In the countryside, $3.67 \%$ of subjects were diagnosed with AD. Interestingly, the rates of patient-reported AD symptoms were significantly higher in urban areas, with over $40 \%$ of children aged 6-7 and 14-15 affected (the symptoms were reported by the children's legal guardians). The proportion of patient-reported AD symptoms in the adult group was 34.91\% [25]. The patient-reported rates illustrate the scale of the problem of dermatoses, especially in the population of younger children. The main causes of skin problems are parabens (esters of 4-hydroxybenzoic acid). These compounds are considered to be among the most common preservatives used in cosmetic products

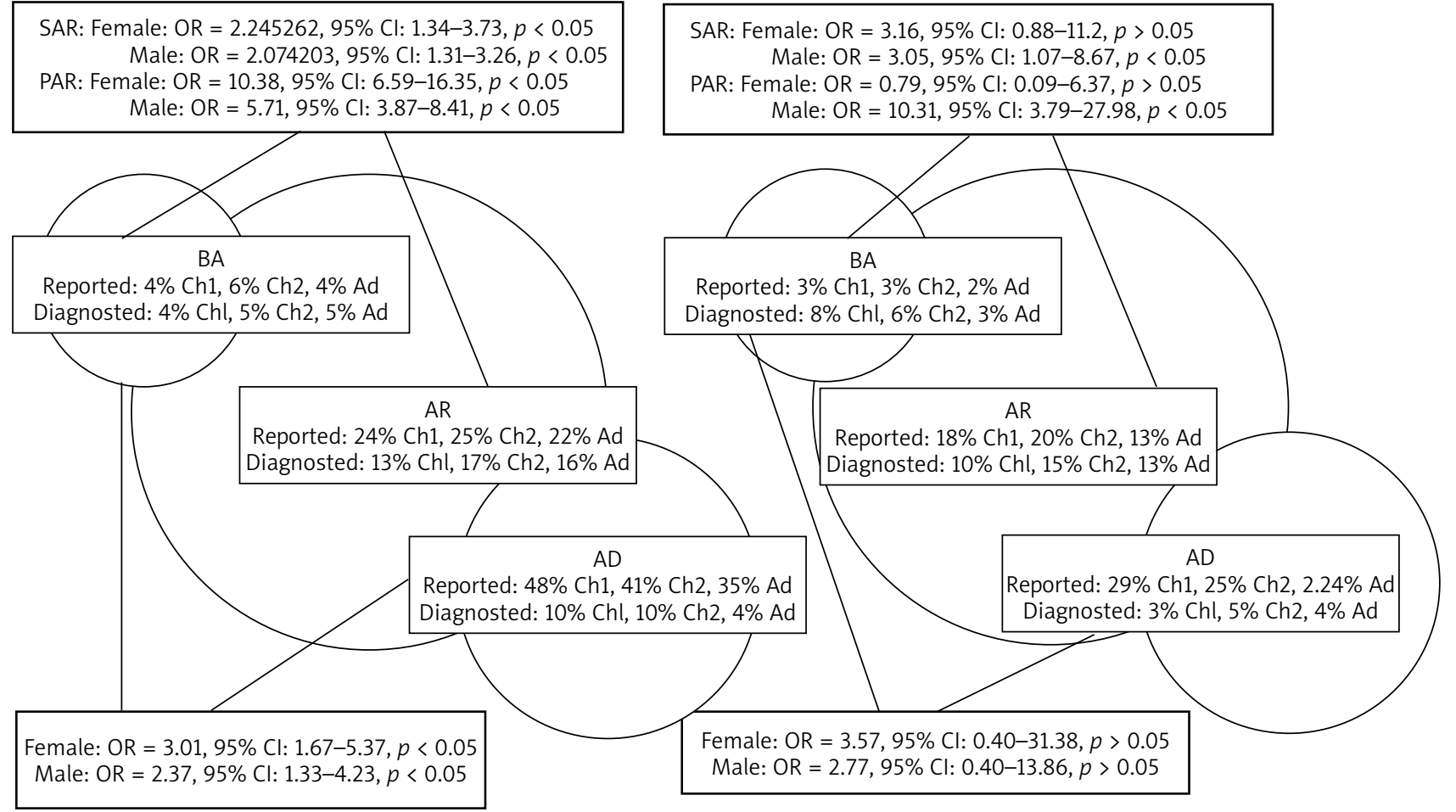

A - Urban areas

B - Countryside

$A D$ - atopic dermatitis, $A d$ - adults, $A R$ - allergic rhinitis, BA - bronchial asthma, Ch1 - children 6-7 years old, Ch2 - children 13-14 years old, $P A R$ - perennial allergic rhinitis, SAR - seasonal allergic rhinitis.

Figure 1. The prevalence of allergic conditions in the questionnaire-based study in urban areas (A) vs. countryside (B) 
(they are found in 22,000 cosmetic products approved for everyday use), food products, and medications [26].

Positive skin tests were observed considerably more often in residents of metropolitan areas. The dominant allergens were seasonal and included grasses/cereals, birch, wormwood, and D. farinae. The group of young children living in the countryside was found to be most commonly affected by house dust mite and dog allergens. In adults, co-allergens were grasses/cereals and wormwood. The phenomenon of patient-reported allergies to house dust mites despite negative skin tests is common among countryside residents [2]. These findings were similar to those obtained by Bousquet et al. in ECRHS I study conducted in 35 centers around the world, Nicolai and von Mutius in Germany, and Sożanska et al. in Poland [27-29].

Genetic etiology of allergic conditions has been confirmed in a number of studies; the risk of a co-occurring atopic condition is $25-35 \%$ in individuals whose sibling has been diagnosed with allergy, 30-50\% when one or both parents are affected by allergies, and $70 \%$ when both parents suffer from the same allergic condition [30]. These findings are consistent with the observed incidence of BA and AR [1]. The last decade saw identification of various human leukocyte antigens (HLA) that play a crucial role in AR [1]. Moreover, based on the presence of filaggrin loss-of-function mutations 2282del4 and R501X (typically predisposing to AD in the Caucasian populations by disrupting keratin fiber binding in the late stages of differentiation and affecting the cornified envelope), Ponińska et al. (ECAP study) estimated a high risk of developing co-occurring allergies in a group of 3,802 subjects with $A D$ $(\mathrm{OR}=2.01,95 \% \mathrm{Cl}:$ 1.20-3.36, $p=0.007), \mathrm{AR}(\mathrm{OR}=1.69$, $95 \% \mathrm{Cl}: 1.12-2.54, p=0.011)$, and $\mathrm{BA}(\mathrm{OR}=2.22,95 \% \mathrm{Cl}$ : $1.24-3.96, p=0.006)$. This suggests that efforts to restore the epidermal barrier may play an important role in BA prevention and treatment [31].

The observed region-dependent increase in the incidence of allergic diseases within the last decade (western lifestyle, increased use of agricultural chemicals in Poland, gradual obliteration of the typical features of the Polish countryside) calls for introducing measures of primary prophylaxis, as well as prevention of complications and secondary allergies. In light of the significant problem of asthma underdiagnosis and the findings of this study, there is a need to initiate preventive measures and implement early diagnosis of rhinologic and allergic disorders.

\section{Acknowledgments}

This study was supported by project 6 PO5 2005 C/06572 "Implementation of a System for the Prevention and Early Detection of Allergic Diseases in Poland" (ECAP, Epidemiology of Allergic Diseases in Poland), as commis- sioned by the Minister of Health (granted to Professor Bolesław Samoliński)

\section{Conflict of interest}

The authors declare no conflict of interest.

\section{References}

1. Samoliński B, Raciborski F, Lipiec A, et al. Epidemiology of allergic diseases in Poland. Pol I Allergol 2014; 1: 10-8.

2. Bousquet J, Khalataev N, Alvaro A, et al. Allergic Rhinitis and its Impact on Asthma (ARIA) 2008. Alergia Astma Immunol Klin 2008; 13: 3-39.

3. Allergic Rhinitis and its Impact on Asthma (ARIA) Revision 2010-V. 9/8/2010 whiar.org/docs/ariareport_2010.pdf

4. Ogino S, Irifune M, Harada T, et al. Nasal allergy in medical students. Rhinology 1990; 28: 163-8.

5. Hagy GW, Settipane GA. Bronchial asthma, allergic rhinitis and allergy skin tests among college students. J Allergy 1996; 44: 323-32.

6. Richards S, Thornhill D, Roberts H, et al. How many people think they have hay fever, and what they do about it. $\mathrm{Br}$ J Gen Pract 1992; 42: 284-6.

7. Huang SK, Zhang Q, Qiu Z, Chung KF. Mechanistic impact of outdoor air pollution on asthma and allergic diseases. J Thorac Dis 2015; 7: 23-33.

8. Zhang Q, Qiu Z, Chung KF, Huang SK. Link between environmental air pollution and allergic asthma: East meets West. J Thorac Dis 2015; 7: 14-22.

9. Son JY, Lee JT, Park YH, Bell ML. Short-term effects of air pollution on hospital admissions in Korea. Epidemiology 2013; 24: $545-54$.

10. D’Amato G, Baena-Cagnani CE, Cecchi L, et al. Climate change, air pollution and extreme events leading to increasing prevalence of allergic respiratory diseases. Multidiscip Respir Med 2013; 8: 12.

11. Kim HH, Lee CS, Jeon JM, et al. Analysis of the association between air pollution and allergic diseases exposure from nearby sources of ambient air pollution within elementary school zones in four Korean cities. Environ Sci Pollut Res Int 2013; 20: 4831-46.

12. D’Amato G, Cecchi L, D’Amato M, Liccardi G. Urban air pollution and climate change as environmental risk factors of respiratory allergy: an update. J Investig Allergol Clin Immunol 2010; 20: 95-102.

13. McCreanor J, Cullinan P, Nieuwenhuijsen MJ, et al. Respiratory effects of exposure to diesel traffic in persons with asthma. N Engl J Med 2007; 357: 2348-58.

14. Wojtyniak B, Piekarski T. 1996-Short term effect of air pollution on mortality in Polish urban population - what is different. J Epidemiol Commun Health 1996; 50 (Suppl. 1): 36-41.

15. Tomaszewska A, Raciborski F, Samel-Kowalik P, Samoliński B. Frequency of allergic rhinitis in selected regions of Poland. City vs countryside analysis. Otolaryngol Pol 2007; 61: 550-3.

16. Tomaszewska A. [The prevalence of allergic diseases in the urban and rural area - comparative analysis]. Doctorat thesis, Warsaw 2014; 75-80.

17. Komorowski J. [Epidemiology of asthma in Poland based on the ECAP study results]. Doctorat thesis, Warsaw 2012; 13-7.

18. Kupryś-Lipińska I, Elgalal, Kuna P. Urban-rural differences in the prevalances of atopic diseases in the general popula- 
tion in Lodz Province (Poland). Postep Derm Alergol 2009; 26: 249-56.

19. Blair H. Natural history of childhood asthma: 20-year followup. Arch Dis Child 1997; 52: 613-9.

20. Settipane GA. Allergic rhinitis-update. Otolaryngol Head Neck Surg 1986; 94: 470-5.

21. Leynaert B, Settipane GA. Long-term risk factors for developing asthma and allergic rhinitis: a 23-year follow-up study of college students. Allergy Proc 1994; 15: 21-8.

22. Worldwide variation in prevalence of symptoms of asthma, allergic rhinoconjunctivitis, and atopic eczema: ISAAC. The International Study of Asthma and Allergies in Childhood (ISAAC). Eur Respir J 1998; 12: 315-35.

23. Variations in the prevalence of respiratory symptoms, selfreported asthma attacks, and use of asthma medication in the European Respirtory Health Survey (ECRHS). Eur Respir J 1996; 9: 687-95.

24. Samoliński B, Sybilski A, Raciborski F, et al. [Prevalence of rhinitis in Polish population according to the ECAP (Epidemiology of Allergic Disorders in Poland) study]. Otolaryngol Pol 2009; 63: 324-30.

25. Sybilski A, Raciborski F, Lipiec A, et al. Atopic dermatitis is a serious health problem in Poland. Epidemiology studies based on the ECAP study. Postep Derm Allergol 2015; 32: $1-10$.

26. Bojarowicz H, Wnuk M, Buciński A. Efficacy and safety of paraben use. Probl Hig Epidemiol 2012; 93: 647-53.

27. Bousquet PJ, Chinn S, Janson C, et al. Geographical variation in the prevalence of positive skin tests to environmental aeroallergens in the European Community Respiratory Health Survey I. Allergy 2007; 62: 301-9.

28. Nicolai T, von Mutius E. Respiratory hypersensitivity and environmental factors: East and West Germany. Toxicol Lett 1996; 86: 105-13.

29. Sożańska B, MacNeill S J, Kajderowicz-Kowalik M, et al. Atopy and asthma in rural Poland: a paradigm for emergence of childhood respiratory allergies in Europe. Allergy 2007; 62: 394-400.

30. Kjellman NI. Atopic disease in seven-year-old children. Incidence in relation to family history. Acta Paediatr Scand 1977; 66: 465-71.

31. Ponińska J, Samoliński B, Tomaszewska A, et al. Filaggrin gene defects are independent risk factors for atopic asthma in a Polish population: a study in ECAP Cohort. PLoS One 2011; 6: e16933. 